\title{
Correlation between carbon flux through the pentose phosphate pathway and production of the antibiotic methylenomycin in Streptomyces coelicolor A3(2)
}

\author{
A. I. C. Obanye, ${ }^{1}$ G. Hobbs, ${ }^{2}$ D. C. J. Gardner ${ }^{1}$ and S. G. Oliver ${ }^{1}$ \\ Author for correspondence: S. G. Oliver. Tel: +44161200 4222. Fax: +441612360409. \\ e-mail: steve.oliver@umist.ac.uk
}

\begin{abstract}
1 Department of Biochemistry and Applied Molecular Biology, UMIST, PO Box 88, Sackville Street, Manchester M60 1QD, UK

2 School of Biomolecular Sciences, Liverpool John Moores University, Byrom Street, Liverpool L3 3AF, UK
\end{abstract}

\begin{abstract}
Radiorespirometry was employed to study carbon metabolism during the growth of Streptomyces coelicolor A3(2) in a minimal medium which permitted the production of methylenomycin as the sole detectable secondary metabolite. A switch in the pattern of carbon metabolism from the Embden-Myerhof-Parnas pathway to the pentose phosphate pathway occurred during the period of slower growth in batch culture which immediately preceded entry into the stationary phase. This coincided with the period of methylenomycin production. It is proposed that the biosynthesis of methylenomycin is supported by the generation of NADPH during the latter part of growth.
\end{abstract}

Keywords: secondary metabolism, pentose phosphate pathway, methylenomycin, Streptomyces coelicolor A3(2), radiorespirometry

\section{INTRODUCTION}

Streptomycetes are filamentous soil bacteria whose life cycle involves the formation of a prostrate mycelium which, following the cessation of vegetative growth, produces aerial hyphae and spores. This period of morphological differentiation is usually accompanied by a change in metabolism toward the synthesis of secondary products which include biologically active molecules, such as antibiotics and herbicides. Although the synthesis of antibiotics and other secondary metabolites is not linked to growth (by definition; Demain, 1992), it may occur in either the growth or the stationary phase of batch culture (Hobbs et al., 1990). The particular growth phase with which secondary product formation is associated depends, to a large extent, upon the nutritional environment in which the producing organism is grown.

Streptomyces coelicolor A3(2) has been adopted as a model system in which it has been possible to identify clusters of genes responsible for the synthesis of, and resistance to, antibiotics (Narva \& Feitelson, 1990; Malpartida et al., 1990; Malpartida \& Hopwood, 1984, 1986). A number of genes which regulate both physiological differentiation (antibiotic production) and morphological differentiation

Abbreviations: EMP, Embden-Meyerhof-Parnas; PP, pentose phosphate; TCA, tricarboxylic acid. (sporulation) have also been defined (Hopwood, 1988). In spite of these achievements, our understanding of the precise physiological changes which trigger secondary product formation, as well as the mechanisms by which metabolism is redirected towards this end, are still rudimentary. Although this species is not the most amenable of subjects for physiological research, systems have been developed which facilitate such experiments (Hobbs et al., 1989) and permit the integration of the molecular genetical and physiological approaches to the study of secondary metabolism (Hobbs et al., 1992).

Methylenomycin is representative of a class of antibiotics possessing cyclopentanone nuclei, of which several have been isolated (McGahren et al., 1969; Umino et al., 1973; Noble et al., 1978). It is derived, principally, from acetylCoA and pyruvate (Hornemann \& Hopwood, 1981). In S. coelicolor A3(2) grown on a minimal medium containing alanine as the sole nitrogen source, glucose as the principal carbon source and high levels of phosphate, methylenomycin is the only secondary product formed and it accumulates during the period of slow growth which marks the transition between the exponential and stationary phases in batch culture (Hobbs et al., 1992). Both pyruvate and 2-oxoglutarate are produced during the acidogenic exponential growth phase of these cultures. The period of methylenomycin production precedes glucose exhaustion, but coincides with the apparent reabsorption of pyruvate from the medium. 


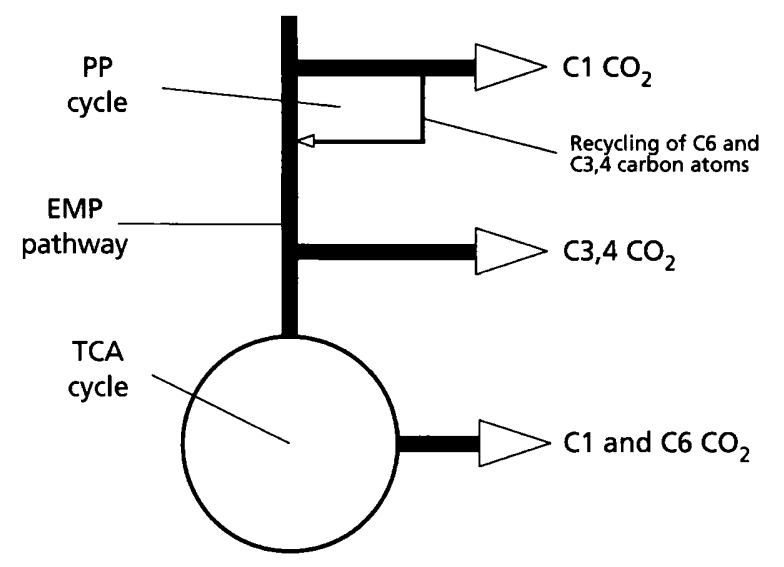

Fig. 1. Rationale of experiments. The ratios investigated in this work were obtained by considering the degree to which the C1, C3,4 and C6 carbon atoms of glucose were released from the respective metabolic pathways in the form of $\mathrm{CO}_{2}$. According to the simplified model presented here, $\mathrm{C1} \mathrm{CO}_{2}$ is released both from the oxidative branch of the PP pathway and from the oxidative processes of the tricarboxylic acid (TCA) cycle. However, as $\mathrm{C} 1$ and $\mathrm{C} 6 \mathrm{CO}_{2}$ are released to the same extent from the TCA cycle (due to the equilibration reaction at the level of triose phosphate isomerase in the EMP pathway), the amount of $\mathrm{CO}_{2}$ released from the oxidative branch of the PP pathway is obtained as the value $\mathrm{C} 1-\mathrm{C} \mathrm{CO}_{2}$. Flux through the TCA cycle is represented by the value $\mathrm{C}_{6} \mathrm{CO}_{2}$, while flux through the EMP pathway $\left(\mathrm{CO}_{2}\right.$ released by oxidation via pyruvate dehydrogenase) is represented by $\mathrm{C}_{3}, 4 \mathrm{CO}_{2}$.

In $S$. coelicolor, little is known about carbon metabolism and how this might alter during the period of antibiotic production. In Streptomyces aureofaciens, DiMarco (1956; quoted in Cochrane, 1961) has suggested that the balance of the Embden-Myerhof-Parnas (EMP) and pentose phosphate (PP) pathways is affected by the phosphate concentration in the medium. He found that the initial phase of growth was dominated by the EMP pathway, while a second phase (during which the medium phosphate concentration was depleted) was typified by an active PP pathway. Chlortetracycline was produced by $S$. aureofaciens during this second phase of growth. Penzikova \& Levitov (1966) have also suggested that the rate of antibiotic biosynthesis in Streptomyces (Actinomyces) fradiae was dependent, to a large extent, upon the nature of flux through the pathways by which carbohydrates are degraded, and that the rate of antibiotic synthesis is regulated by carbon metabolism. Primary metabolism and antibiotic production have been correlated in a number of other streptomycetes (Hostalek, 1969; Vorisek et al., 1969; Thiericke \& Zeeck, 1988; Dekleva \& Strohl, 1988a, b; Canovas \& Kornberg, 1965). Particularly relevant is the discovery by Ikeda et al. (1988) that the activity of 6phosphogluconate dehydrogenase of the PP pathway was correlated with avermectin production in Streptomyces avermitilis. These investigators emphasized the importance of this enzyme in providing NADPH for avermectin biosynthesis.

In this study we report on the balance between the EMP and PP pathways of carbon catabolism in S. coelicolor
A3(2) during batch and continuous culture, using an adaptation of the radiorespirometric methodology of Wang et al. (1958). The rationale governing this approach is explained in Fig. 1.

\section{METHODS}

Bacterial strain. S. coelicolor A3(2) strain 1147 was used throughout the project.

Culture conditions. All experiments were carried out using Hobbs' minimal medium (HMM; Hobbs et al., 1989), adapted for the exclusive synthesis of methylenomycin by the substitution of alanine for nitrate as the sole source of nitrogen (Hobbs et al., 1992). Methods for inoculum preparation and fermentation conditions in small shake flasks and 21 bioreactors were as described by Hobbs et al. $(1989,1992)$.

Sampling procedures. Samples $(1 \mathrm{ml})$ were withdrawn from shake flasks or fermenters and the $\mathrm{OD}_{550}$ was determined. Further aliquots $(1 \mathrm{ml})$ were centrifuged in a microcentrifuge, after which the supernatant and the cell pellet were separated and both stored at $-20^{\circ} \mathrm{C}$.

Radiorespirometric analysis. The metabolism of exogenously supplied glucose was monitored using the following radiorespirometric technique. Duplicate aliquots $(1 \mathrm{ml})$ were withdrawn from the same $250 \mathrm{ml}$ conical flask as used for biomass and supernatant characterization, and placed in a $15 \mathrm{ml}$ capacity glass vial (Aldrich) which contained the radioactive metabolite (C1- and C6-labelled glucose, Amersham; C3,4labelled glucose, Dupont NEN Research). The final vial concentration of C3,4-labelled glucose was $0.0019 \mathrm{mM}$ and its specific activity was $10 \cdot 3 \mathrm{mCi} \mathrm{mmol}^{-1}\left(381 \mathrm{MBq} \mathrm{mmol}^{-1}\right)$. The respective values for $\mathrm{C} 1$ - and $\mathrm{C} 6$-labelled glucose were $0.0036 \mathrm{mM}^{2}$ and $55.8 \mathrm{mCi} \mathrm{mmol}^{-1}\left(2064 \mathrm{MBq} \mathrm{mmol}^{-1}\right)$. The vial was sealed with a rubber stopper (Fisons Scientific Apparatus) through which was suspended a Konte centre well (Burkard Scientific) containing a filter paper plug. Vials were incubated at $30^{\circ} \mathrm{C}$ for $40 \mathrm{~min}$ in a shaking water bath (Infors). Incubation was terminated by injecting $150 \mu \mathrm{l}$ of $1 \mathrm{M} \mathrm{HCl}$ through the rubber cap into the culture. The $\mathrm{CO}_{2}$ released was trapped by injecting $300 \mu \mathrm{l}$ of a $1: 1$ mixture of $\beta$-phenylethylamine/ methanol into the Konte well. Trapping was conducted for $1 \mathrm{~h}$. The incubation and trapping time, as well as the amounts of trapping agent and $\mathrm{HCl}$ required, were established in separate experiments (Obanye, 1994). After the $\mathrm{CO}_{2}$ had been trapped, the Konte centre well and filter paper were transferred to $4 \mathrm{ml}$ scintillation cocktail (Optiphase 'Hi-Safe' 3 ; Wallac) in a plastic scintillation vial (Sterilin). This was subsequently analysed using an LKB Wallac 1216 Rackbeta II liquid scintillation counter. The window settings were $50-165$ and the counts were corrected to d.p.m. using the external standard channels ratio method (Herberg, 1963). $\mathrm{CCl}_{4}$ was used as the quenching agent. The accuracy of counting lay within $\pm 5 \%$.

Metabolic assays. Concentrations of organic acids, glucose and methylenomycin in the culture supernatants were measured as described previously (Hobbs et al., 1992).

\section{RESULTS}

\section{Variation of radiorespirometric ratios during batch growth}

In batch culture, during the exponential phase of growth, there was a fall in the $\mathrm{C} 1-\mathrm{C} 6 / \mathrm{C} 3,4$ ratio (i.e. [flux through the PP pathway]/[flux through the EMP pathway]) to a 


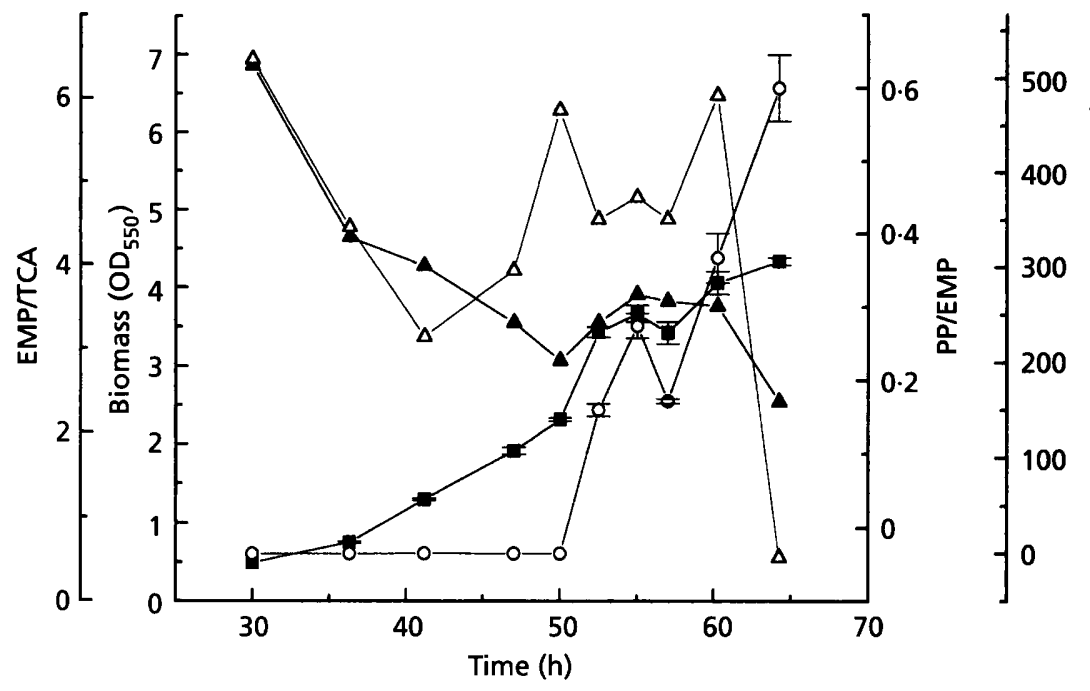

Fig. 2. Radiorespirometric analysis of Cmetabolism in batch culture. Biomass $(\square)$ and methylenomycin (O) concentrations and the radiorespirometric ratios: PP/EMP $(\triangle)$ and EMP/TCA $(\boldsymbol{A})$. Values shown are the mean of two measurements; the bars indicate the range of values.

Table 1. Minimum and maximum values of PP/EMP and EMP/TCA

\begin{tabular}{|c|c|c|c|c|c|c|c|c|}
\hline Expt ... & 1 & 2 & 3 & 4 & 5 & 6 & 7 & Mean (SD) \\
\hline $\mathrm{A}=\mathrm{Min} .[\mathrm{PP} / \mathrm{EMP}]$ & $0 \cdot 26$ & $0 \cdot 19$ & $0 \cdot 16$ & $0 \cdot 14$ & $0 \cdot 35$ & $0 \cdot 33$ & $0 \cdot 20$ & $0.23(0.08)$ \\
\hline $\mathrm{B}=\mathrm{Max} .[\mathrm{PP} / \mathrm{EMP}]$ & 0.59 & 0.93 & $1 \cdot 64$ & 0.74 & $1 \cdot 02$ & $1 \cdot 74$ & $1 \cdot 13$ & $1 \cdot 11(0 \cdot 43)$ \\
\hline $\mathrm{C}=\mathrm{Min} .[\mathrm{EMP} / \mathrm{TCA}]$ & 3.07 & $2 \cdot 73$ & $2 \cdot 12$ & $2 \cdot 97$ & $2 \cdot 81$ & $2 \cdot 86$ & $2 \cdot 91$ & $2 \cdot 78(0 \cdot 31)$ \\
\hline $\mathrm{D}=\mathrm{Max} .[\mathrm{EMP} / \mathrm{TCA}]$ & 3.91 & $3 \cdot 43$ & $4 \cdot 42$ & 3.92 & 3.93 & 3.64 & $5 \cdot 62$ & $4 \cdot 12(0.73)$ \\
\hline $\mathrm{B} / \mathrm{A}$ & $2 \cdot 27$ & $4 \cdot 98$ & $10 \cdot 12$ & $5 \cdot 29$ & $2 \cdot 91$ & $5 \cdot 27$ & $5 \cdot 65$ & $5 \cdot 21(2 \cdot 53)$ \\
\hline $\mathrm{D} / \mathrm{C}$ & $1 \cdot 27$ & $1 \cdot 26$ & $2 \cdot 08$ & $1 \cdot 32$ & $1 \cdot 37$ & $1 \cdot 27$ & 1.93 & $1.50(0 \cdot 35)$ \\
\hline
\end{tabular}

* $\mathrm{A}$ is the minimum value that the PP/EMP ratio attains during exponential growth. $\mathrm{B}$ is the maximum value that the $\mathrm{PP} / \mathrm{EMP}$ ratio attains just prior to, or during, the production of methylenomycin. $\mathrm{C}$ is the minimum value of the EMP/TCA ratio attained prior to the production of methylenomycin. $\mathrm{D}$ is the maximum value of the EMP/TCA ratio attained at or around the onset of methylenomycin production.

minimum value at the mid-exponential phase (Fig. 2). The value of this ratio at its minimum varied from $0 \cdot 14$ to 0.35 (Table 1). After this point, this ratio rose again to achieve a maximum at or around the point at which growth slowed and methylenomycin production was initiated. During this slow growth phase, the PP/EMP ratio remained elevated. The value of the maximum during the latter stages of growth varied from between about 0.6 and 1.74 in six replicate experiments.

From these results, it is apparent that the carbon flux through the PP cycle was less than that passing through the EMP pathway during the exponential growth phase. When the specific growth rate had slowed, and methylenomycin was being produced, relative flux through the PP pathway increased and, in some experiments, exceeded that through the EMP pathway. However, the variation observed between replicate experiments suggests that the data provide only a qualitative picture of the balance of carbon flux between the two pathways.

With regard to the specific rates of production of $\mathrm{C} 1-\mathrm{C} 6$ $\mathrm{CO}_{2}$ (PP flux; mmol per $\mathrm{OD}_{550}$ unit $\mathrm{min}^{-1}$ ) and of $\mathrm{C} 6 \mathrm{CO}_{2}$ (TCA flux; mmol per $\mathrm{OD}_{550}$ unit $\mathrm{min}^{-1}$ ), the increases in the $\mathrm{PP} / \mathrm{EMP}$ ratio during the production of methyleno- mycin appeared to be associated predominantly with a real increase in the PP flux rather than a fall in the TCA flux (Table 2). The other important metabolic parameter was the EMP/TCA ratio, which was also seen to fall during the exponential phase, reaching a minimum value at or around the time that the $\mathrm{PP} / \mathrm{EMP}$ ratio attained its maximum (Fig. 2). The value of the EMP/TCA ratio, at its minimum, varied between 2.12 and 2.97 in six replicate experiments (Table 1). Thereafter, the ratio rose again during the production phase, attaining a maximum of between 3.64 and 5.62 (Table 1). Thus not only was there a real increase in the specific rate of $\mathrm{C} 1-\mathrm{C}_{6} \mathrm{CO}_{2}$ production (mmol per $\mathrm{OD}_{550}$ unit $\mathrm{min}^{-1}$ ), representing a real increase in PP flux, but there was also an increase in the specific rate of $\mathrm{C} 3,4 \quad \mathrm{CO}_{2}$ production (flux through pyruvate dehydrogenase; mmol per $\mathrm{OD}_{550}$ unit $\mathrm{min}^{-1}$; Fig. 2). This latter observation is not shown in Table 2, which simply compares the two time points immediately before the onset of methylenomycin production with those immediately after this point.

The highest values of the PP/EMP ratio were found during the periods of slowest growth; however experiments using continuous-flow cultures (data not shown) 
Table 2. Mean rate of $\mathrm{CO}_{2}$ release over the two time points subsequent to the onset of methylenomycin production relative to the two time points prior to methylenomycin production, from six independent experiments

\begin{tabular}{|c|c|c|c|}
\hline \multirow[t]{2}{*}{ Expt } & \multicolumn{3}{|c|}{ Change in mean rate of $\mathrm{CO}_{2}$ release $(\%)^{*}$} \\
\hline & C1-C6 $\mathrm{CO}_{2}$ & $\mathrm{C} 6 \mathrm{CO}_{2}$ & $\mathrm{C} 3,4 \mathrm{CO}_{2}$ \\
\hline 1 & $152\left(4.7 \times 10^{-3}\right)$ & $229\left(2 \cdot 1 \times 10^{-3}\right)$ & $203\left(6.3 \times 10^{-3}\right)$ \\
\hline 2 & $115\left(2 \cdot 7 \times 10^{-3}\right)$ & $94\left(9 \cdot 7 \times 10^{-4}\right)$ & $96\left(3.3 \times 10^{-3}\right)$ \\
\hline 3 & $224\left(1.2 \times 10^{-2}\right)$ & $101\left(2 \cdot 8 \times 10^{-3}\right)$ & $99\left(9 \cdot 1 \times 10^{-3}\right)$ \\
\hline 4 & $375\left(4.7 \times 10^{-3}\right)$ & $79\left(1 \cdot 0 \times 10^{-3}\right)$ & $123\left(5.5 \times 10^{-3}\right)$ \\
\hline 5 & $266\left(3.6 \times 10^{-3}\right)$ & $125\left(1.2 \times 10^{-3}\right)$ & $67\left(4.0 \times 10^{-3}\right)$ \\
\hline 6 & $133\left(4 \cdot 0 \times 10^{-3}\right)$ & $125\left(2.5 \times 10^{-3}\right)$ & $142\left(9 \cdot 2 \times 10^{-3}\right)$ \\
\hline $\begin{array}{l}\text { Mean } \\
\quad(n=6)\end{array}$ & $210\left(3.5 \times 10^{-3}\right)$ & $126\left(1.8 \times 10^{-3}\right)$ & $122\left(6 \cdot 2 \times 10^{-3}\right)$ \\
\hline $\begin{array}{l}\mathrm{SD} \\
\quad(n-1)\end{array}$ & $99\left(1.3 \times 10^{-3}\right)$ & $49\left(8.1 \times 10^{-4}\right)$ & $47\left(2.5 \times 10^{-3}\right)$ \\
\hline
\end{tabular}

* The quantitive value of the mean rate of $\mathrm{CO}_{2}$ released $(\mathrm{mmol}$ per $\mathrm{OD}_{550}$ unit $\mathrm{min}^{-1}$ ) in each case is shown in parentheses.

established that specific growth rate itself was not responsible for the variations in both ratios.

\section{DISCUSSION}

The use of radiorespirometry demonstrated that significant changes in carbon catabolism occurred around the time at which methylenomycin was produced. In this work, this was observed as the result of ordinary batch growth processes. A similar switch in glucose metabolism was seen as a consequence of artificially lowering the medium $\mathrm{pH}$ in a chemostat (Obanye, 1994). The most important change observed was a significant increase in the flux through the oxidative limb of the pentose phosphate pathway.

All phases of growth were associated with the presence of organic acids in the culture medium (Hobbs et al., 1992). Organic acid production by micro-organisms is a common phenomenon. 2-Oxoglutarate and pyruvate are the ketoacids most commonly reported to be produced by streptomycetes (Ahmed et al., 1984). In our own experiments, it was during the slow transition phase that the medium concentration of 2-oxoglutarate stabilized while that of pyruvate declined. The latter event suggested active reassimilation of pyruvate and, possibly, the utilization of this organic acid for incorporation into methylenomycin (Hornemann \& Hopwood, 1981). Pyruvate is also a gluconeogenic substrate. Such changes in acidogenesis intimate either metabolic or transport changes occurring during the change in the growth phase. Although transport studies were not carried out, it is likely that the excretion of organic acids was dependent on a combination of transport and metabolic factors.

Our radiorespirometric results suggest that there may be a relationship between the regulation of methylenomycin production and the carbon catabolic changes that occur throughout the latter part of the growth cycle of $S$. coelicolor A3(2). This relationship is demonstrated by Table 2, in which the mean values of the specific rates of $\mathrm{CO}_{2}$ released from the PP pathway, the TCA cycle, and the EMP pathway immediately after the onset of methylenomycin production are compared with those recorded just prior to the production of methylenomycin, for six independent experiments. The production phase is accompanied by an increased rate of glucose catabolism in all cases, the greatest effect being seen for $\mathrm{CO}_{2}$ released via the PP pathway. We suggest that the relationship between the regulation of methylenomycin production and the carbon catabolic changes observed at or around the start of the the production phase involves the utilization of NADPH, generated by the flux through the PP pathway.

Many antibiotics, notably those derived from polyketides, are synthesized reductively and the operation of pathways that generate NADPH is often essential to secondary metabolite production (Ikeda et al., 1988). In all our experiments, the bulk of methylenomycin production occurred after a real elevation in the rate of flux through the PP pathway had taken place. The other important parameter was the EMP/TCA ratio (i.e. the C3,4/C6 ratio). It may be significant that this ratio was seen to rise at approximately the same time that methylenomycin was first detected in the medium, at a point where the increased generation of NADPH was probably crucial, and during a period where the concentration of pyruvate was falling. The tendency for the decrease in the pyruvate concentration to coincide with an increase in the EMP/TCA ratio supports the idea that there was an increase in flux through pyruvate dehydrogenase, perhaps due to the reduction of the NADH/NAD ${ }^{+}$ratio. Such a reduction might have arisen from the combination of a fall in the extracellular $\mathrm{pH}$ and utilization of $\mathrm{NADH}$ in a transhydrogenase reaction involved in the generation of NADPH either via the operation of nicotinamide nucleotide transhydrogenase (Yamaguchi \& Hatefi, 1991; Yamaguchi et al., 1988; Ahmad et al., 1992) or indirectly via coupled enzyme systems.

It is not known definitively whether a supply of NADPH is necessary for the production of methylenomycin. An increase in the EMP/TCA ratio would be consistent with a flow of carbon into acetyl-CoA in that the flux from the EMP pathway did not seem to be transmitted to the TCA cycle to a proportionate extent. Acetyl-CoA is an important precursor for the synthesis of methylenomycin (Hornemann \& Hopwood, 1981) and polyketides alike. In the latter case, it is known that NADPH plays a crucial part in this process (Herbert, 1989).

\section{ACKNOWLEDGEMENTS}

This work was supported by a SERC studentship to A.I.C.O. and by grants from SERC and the SERC/DTI/Glaxo Wellcome/SmithKline Beecham Link initiative to S.G.O. and C. P. Smith. We would also like to thank SmithKline Beecham and Glaxo Wellcome for additional financial support to A.I.C.O. We are grateful to Dr Phil Butler for his critical appraisal of the manuscript. 


\section{REFERENCES}

Ahmad, S., Glavas, N. A. \& Bragg, P. D. (1992). A mutation at Gly 314 of the $\beta$ subunit of the Escherichia coli pyridine nucleotide transhydrogenase abolishes activity and affects the NADP $(\mathrm{H})$ induced conformational change. Eur J Biochem 207, 733-739.

Ahmed, Z. A., Shapiro, S. \& Vining, L. C. (1984). Excretion of $\alpha$ keto acids by strains of Streptomyces venezuelae. Can J Microbiol $\mathbf{3 0}$, 1014-1021.

Canovas, J. L. \& Kornberg, H. L. (1965). Fine control of phosphopyruvic carboxylase activity in Escherichia coli. Biochim Biopbys Acta 96, 169-172.

Cochrane, V. W. (1961). Physiology of actinomycetes. Annu Rev Microbiol 15, 1-26.

Dekleva, M. L. \& Strohl, W. R. (1988a). Biosynthesis of $\varepsilon$-rhodomycinone from glucose by Streptomyces $\mathrm{C} 5$ and comparison with intermediary metabolism of other polyketide-producing streptomycetes. Can J Microbiol 34, 1235-1240.

Dekleva, M. L. \& Strohl, W. R. (1988b). Activity of phosphoenolpyruvate carboxylase of an anthracycline-producing streptomycete. Can J Microbiol 34, 1241-1246.

Demain, A. L. (1992). Microbial secondary metabolism: a new theoretical frontier for academia, a new opportunity for industry. In Secondary Metabolites: Their Function and Evolution, pp. 3-23. Edited by D. J. Chadwick \& J. Whelan. Chichester: John Wiley.

Herberg, R. J. (1963). Statistical aspects of liquid scintillation counting by internal standard techniques. Single isotope. Anal Chem 35, 786-791

Herbert, R. B. (1989). The Biosynthesis of Secondary Metabolites, 2nd edn. London: Chapman \& Hall.

Hobbs, G., Frazer, C. M., Gardner, D. C. J., Cullum, J. A. \& Oliver, S. G. (1989). Dispersed growth of streptomyces in liquid culture. Appl Microbiol Biotechnol 31, 272-277.

Hobbs, G., Frazer, C. M., Gardner, D. C. J., Flett, F. \& Oliver, S. G. (1990). Pigmented antibiotic production by Streptomyces coelicolor A3(2): kinetics and the influence of nutrients. J Gen Microbiol 136, 2291-2296.

Hobbs, G., Obanye, A. I. C., Petty, J., Mason, J. C., Barratt, E., Gardner, D. C. J., Flett, F., Smith, C. P., Broda, P. \& Oliver, S. G. (1992). An integrated approach to studying regulation of production of the antibiotic methylenomycin by Streptomyces coelicolor A3(2). J Bacteriol 174, 1487-1494.

Hopwood D. A. (1988). Towards an understanding of gene switching in Streptomyces, the basis of sporulation and antibiotic production. Proc R Soc Lond B Biol Sci 235, 121-138.

Hornemann, U. \& Hopwood, D. A. (1981). Biosynthesis of methylenomycin A : a plasmid-determined antibiotic. In Antibiotics: Biosynthesis, vol. IV, pp. 123-131. Edited by J. W. Corcoran. New York: Springer-Verlag.

Hostalek, Z. (1969). Regulation of biosynthesis of secondary metabolites. I. Biosynthesis of chlortetracycline and tricarboxylic acid activity. Biotechnol Bioeng 11, 539-548.
Ikeda, H., Kotaki, H., Tanaka, H. \& Omura, S. (1988). Involvement of glucose catabolism in avermectin production by Streptomyces. Antimicrob Agents Chemother 32, 282-284.

McGahren, W. J., van den Hende, J. H. \& Mitscher, L. A. (1969). Chlorinated cyclopentanone fungitoxic metabolites from the fungus, Sporormia affinis. J Am Chem Soc 91, 157-167.

Malpartida, F. \& Hopwood, D. A. (1984). Molecular cloning of the whole biosynthetic pathway of a Streptomyces antibiotic and its expression in a heterologous host. Nature 309, 462-464.

Malpartida, F. \& Hopwood, D. A. (1986). Physical and genetic characterisation of the gene cluster for the antibiotic actinorhodin in Streptomyces coelicolor A3(2). Mol \& Gen Genet 205, 66-73.

Malpartida, F., Niemi, J., Navarette, R. \& Hopwood, D. A. (1990). Cloning and expression in a heterologous host of the complete set of genes for biosynthesis of the Streptomyces coelicolor antibiotic undecylprodigiosin. Gene 93, 91-99.

Narva, K. E. \& Feitelson, J. S. (1990). Nucleotide sequence and transcriptional analysis of the redD locus of Streptomyces coelicolor A3(2). J Bacteriol 172, 326-333.

Noble, M., Noble, D. \& Fletton, R. A. (1978). G2201-C, a new cyclopentenedione antibiotic, isolated from the fermentation broth of Streptomyces cattleya. J Antibiot 31, 15-18.

Obanye, A. (1994). Carbon flux and the production of metbylenomycin in Streptomyces coelicolor A3(2). PhD thesis, University of Manchester.

Penzikova, G. A. \& Levitov, M. M. (1966). Patterns of carbohydrate metabolism of Actinomyces fradiae grown on media containing starch or glucose. Biokbimiya 31, 1073-1077.

Thiericke, R. \& Zeeck, A. (1988). Biosynthesis of manumycin: origin of the polyene chains. J Antibiot 41, 694-696.

Umino K., Furumai, T., Matsuzawa, N., Yamaguchi, Y., Itoh, Y. \& Okuda, T. (1973). Studies on pentenomycins. I. Production, isolation and properties of pentenomycins I and II, new antibiotics from Streptomyces eurothermus MCRL 0738. J Antibiot 26, 506.

Vorisek, J., Powell, A. J. \& Vanek, Z. (1969). Regulation of biosynthesis of secondary metabolites. IV. Purification and properties of phosphoenolpyruvate carboxylase in Streptomyces aureofaciens. Folia Microbiol 14, 398-405.

Wang, C. H., Stern, I., Gilmour, C. M., Klungsoyr, S., Reed, D. J., Bialy, J. J., Christensen, B. E. \& Cheldelin, V. H. (1958). Comparative study of glucose catabolism by the radiorespirometric method. J Bacteriol 76, 207-216.

Yamaguchi, M. \& Hatefi, Y. (1991). Mitochondrial energy-linked nicotinamide nucleotide transhydrogenase: membrane topography of the bovine enzyme. $J$ Biol Chem 266, 5728-5735.

Yamaguchi, M., Hatefi, Y., Trach, K. \& Hoch, J. A. (1988). The primary structure of the mitochondrial energy-linked nicotinamide transhydrogenase deduced from the sequence of cDNA clones. $J$ Biol Chem 263, 2761-2767.

Received 13 July 1995; revised 11 September 1995; accepted 19 September 1995. 